\title{
LAS MAESTRAS EN LA REGIÓN SUR DE COLOMBIA: SIGLO XIX
}

\author{
Gabriela Hernández* \\ Universidad de Nariño - Colombia \\ gabve2003@gmail.com
}

Recepción: 05/04/2011

Evaluación: $15 / 06 / 2011$

Aceptación: 26/08/2011

Artículo de Reflexión

doi: 10,9757

\section{RESUMEN}

El artículo pretende evidenciar la acción pedagógica que realizaron tres maestras a finales del Siglo XIX en las ciudades de Pasto, Ipiales y Túquerres, ubicadas en la región sur del país. El trabajo de estas maestras se desarrolló durante los últimos años del gobierno liberal radical e inicios de la regeneración, razón por la cual, tanto las instituciones educativas como las maestras tuvieron que convivir con las tensiones políticas de la época.

Años en los que, por efectos de la política educativa liberal, fue posible la formación de maestras y maestros mediante la organización de las Escuelas Normales en el país y en particular en Popayán, capital del estado Soberano del Cauca del que por esa época dependía la educación de la región sur de Colombia.
El Proyecto educativo liberal, a pesar de desarrollarse en un contexto de subordinación y exclusión política de las mujeres, creó condiciones para la formación profesional de las maestras quienes, a su vez, impulsaron la cualificación de las escuelas de niñas con planes educativos cuyos objetivos superaron la educación femenina centrada en las primeras letras, moral, educación religiosa y urbanidad.

El artículo corresponde a una parte de un tema más amplio sobre la educación femenina en Pasto $^{1}$, estudio que se realizó teniendo en cuenta la historia social de la región con una posibilidad analítica basada en el género, la clase social y la etnia.

Palabras clave: Revista Historia de la Educación Latinoamericana, maestras decimonónicas- acción pedagógica-educación liberal.

* Doctora en Investigaciones Feministas, Profesora de la Facultad de Educación, Universidad de Nariño. Miembro del Grupo de Investigación reconocido por COLCIENCIAS: Universidad de Nariño: Historia, Educación y Desarrollo. 


\section{FEMALE EDUCATORS IN THE COLOMBIA'S SOUTH REGION: 19th CENTURY}

\begin{abstract}
This article approach as an evidence of teaching activities performed by three female educators at the cities of Pasto, Ipiales y Túquerres all located in the south part of Colombia, on the last part of 19th Century. These Educators' work was developed during the last years of a radical liberal Government period and thorough its recovery era, therefore, all Education Institutes as well as educators and teachers had to survive among political tension and issues of the era.
\end{abstract}

Those were years in which because of the liberal education politics it was posible to instruct both male and female educators thanks to the introduction of Tutoring schools in the country specially in Popayan, Capital city of Cauca Reign State, that was source of education in the south región of Colombia.

The Liberal Educational Project, made possible conditions for the teaching and apprenticeship for women despite of being developed in a context of female discrimination and high command from male politicians. These female educators worked at the same time for improving quality of girl schools with educational plans which had objectives that pull female education focused on first vowels, morals, education on religion and social behavior.

This article is related to a wider subject about femenine education in Pasto ${ }^{1}$ prior study that was developed having in mind all regional social with an unique possibility of understanding an analyses based on gender, social class and ethnicity.

Key words: Journal of Latin American Education History, female educators of 19 th century, pedagogical activism, liberal education.

\section{AS PROFESSORAS NA REGIÃO SUL DA COLÔMBIA: SÉCULO XIX}

\section{RESUMO}

$\mathrm{O}$ artigo pretende evidenciar a ação pedagógica realizada por três professoras no final do século XIX nas cidades de Pasto, Ipiales e Túquerres, localizadas na região sul do país. O trabalho destas professoras se desenvolver durante os últimos anos do governo liberal radical e meados da regeneração, razão pela qual, tanto as instituições educativas como as professoras tiveram que conviver com as tensões políticas da época.

Anos em que, em consequência da política educativa liberal, foi possível a formação de professoras e professores mediante a organização das Escolas Normais no país e em particular em Popayán, capital do estado Soberano de Cauca, de que nessa época dependia a educação da região sul da Colômbia.

O projeto educativo liberal, apesar de desenvolver-se em um contexto de subordinação e exclusão política das mulheres, criou condições para a transformação profissional das professoras, que por sua vez impulsionaram a qualificação das escolas de meninas com planos educativos cujos objetivos superaram a educação feminina centrada nas primeiras letras, moral, educação religiosa e urbanidade.

O artigo corresponde a uma parte de um tema mais amplo sobre a educação feminina em Pasto ${ }^{1}$, estudo realizado a partir da perspectiva da história social da região com uma possibilidade analítica baseada em gênero, classe social e etnia.

Palavras-chave: Revista História da Educação Latino-americana, professoras decimonónicas, ação pedagógica, educação liberal.

\section{INTRODUCCIÓN}

En América Latina la organización de Escuelas Normales para la formación de maestras es un hecho que se presenta a mediados del siglo XIX como efecto de la presencia de gobiernos liberales en la región, así como por la presión de parte de las mujeres que veían limitada su existencia al funcionamiento del hogar y quienes no se conformaban con ser simplemente el "bello sexo" o las "reinas del hogar", ya que ellas sabían de sus posibilidades intelectuales.

\footnotetext{
Gabriela Hernández Vega, “Avatares de la educación femenina en Pasto (Colombia): 1880-1935”. (Tesis doctoral presentada en la Universidad Pablo de Olavide de Sevilla, 2008).
} 
La preocupación por adelantar la consolidación de las nuevas repúblicas llevó a que los gobiernos vieran la necesidad de brindar una educación más amplia a sectores de la población femenina por ser quienes iban a tener la responsabilidad de formar a los futuros ciudadanos. Propósito que no cubrió a la totalidad de las mujeres latinoamericanas, al contrario, se caracterizó por ser expresión de una política bastante excluyente de la que no participaron ni las mujeres indígenas ni las mujeres descendientes de los sectores de esclavos traídos de África.

En Colombia, para la época Estados Unidos de Colombia, las Escuelas Normales Femeninas surgieron en la segunda mitad del siglo XIX como resultado de la política educativa liberal radical, lo que permitió organizar una de estas instituciones en la capital de cada uno de los nueve Estados que para la época conformaban el país. De la región sur, hoy Departamento de Nariño, se graduaron como normalistas solamente tres maestras, no porque no existiera interés de las familias y de las jóvenes por su educación sino por las distancias que dificultaron el acceso a la Escuela Normal de la ciudad de Popayán. Pero, estas tres mujeres fueron suficientes para que, una vez graduadas, impulsaran un verdadero cambio en la educación de las niñas.

\section{Una mirada previa}

La Ilustración europea negó a las mujeres participar de la razón, que fue definida como una cualidad exclusiva del varón. A su vez, ilustrados del país compartieron este concepto: las colombianas al igual que sus semejantes europeas eran pura sensibilidad, motivo para que no fueran consideradas "sujeto de Las Luces, sino que, definida como Naturaleza, es más bien el objeto que Las Luces deben iluminar"”2.

Desde esa perspectiva y en contraste con los varones, la luz que brinda la educación se dio para las mujeres en un contexto en donde primaba para ellas la exclusión de lo público así como la subordinación y sometimiento en el ámbito doméstico. En consecuencia, el impulso de la educación formal para las niñas y las jóvenes no tuvo un propósito diferente el hacer de ellas mejores amas de casa:

\section{Mayor amor y dedicación a los hijos; realizar las labores del hogar con orden y disciplina, saber administrar la casa y a los "sirvientes", convertirse en esposas compasivas y amenas con sus maridos y, además, si les sobraba tiempo utilizarlo para rezar y educarse en labores femeninas para estar preparadas por si faltaba el jefe del hogar ${ }^{3}$.}

2 Cristina Molina Petit, Dialéctica feminista de la Ilustración, (Barcelona: Anthropos, Madrid, Comunidad de Madrid. Consejería de Educación. Dirección General de la Mujer, 1994), 120.

3 Suzy Bermúdez, "Familias y hogares en Colombia durante el siglo XIX y comienzos del XX", en Las Mujeres en la Historia de Colombia. Tomo II. Mujeres y sociedad, Consejería presidencial para la política social, Presidencia de la República, (Santafé de Bogotá: Grupo Editorial Norma, 1995), 248-249. 


\section{La primera maestra en la ciudad de Pasto}

Para los países de la Gran Colombia la pregunta sobre si se debía o no educar a las mujeres fue definida desde 1821 durante la realización del congreso de Cúcuta ${ }^{4}$. Entre los delegatarios no hubo oposición al respecto, estuvieron de acuerdo en el límite de esa educación: formarlas para que fueran buenas esposas y buenas madres, lo que consideraron "muy importante

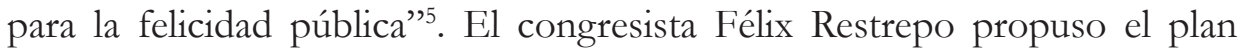
de estudios con el cual se podía atender un programa para la educación femenina, "Que no otra cosa nos conviene desear por ahora sino que las

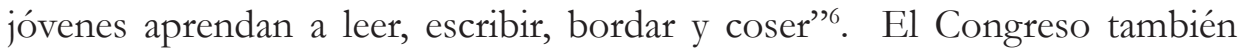
aprobó en una primera resolución de la sesión del 28 de julio que "Artículo $1^{\circ}$. Se establecerán escuelas o casas de educación para las niñas y para las jóvenes en todos los conventos de religiosas"'. Posteriormente, en el mes de agosto se aprobó la Ley 8 sobre creación de escuelas de primeras letras, la cual autorizó fundar escuelas de niñas en las cabeceras de los cantones.

A pesar de ser una época en la cual la educación de las niñas no era aún parte de la cultura cotidiana de la sociedad colombiana debido a que "la mayoría de los padres de familia no le daban importancia a la educación de sus hijas", sino que dentro de sus intereses ocupaban un lugar de privilegio la educación de los niños y de los jóvenes, sin embargo, en Pasto algunas familias interesadas mostraron preocupación por la escolaridad de las niñas la cual fue expresada ante el Concejo Municipal. Como respuesta en el año de 1835 se organizó una Escuela de Primeras Letras para las niñas de la ciudad, y nombraron a quien vendría a ser la primera maestra en una institución educativa formal. Este hecho pasó a ser el punto de partida para que señoritas y señoras de la ciudad se dedicaran al magisterio:

El Concejo Municipal de Pasto en uso de las atribuciones que le concede el articulo 172 de la ley del $1^{\circ}$. De mayo del año pasado,

\section{Considerando:}

$1^{\circ}$. Que el establecimiento de una escuela de niñas es de absoluta necesidad para el adelantamiento del cantón y reforma del sexo cuy a disposición tanto para las letras, como para la costura y demás oficios que les son propios es altamente laudable.

$4 \quad$ Congreso realizado en la ciudad de Cúcuta, el cual dio lugar a la Carta fundacional de la República de la Gran Colombia formada por la Nueva Granada (Colombia y Panamá), Venezuela y Ecuador.

5 Actas del Congreso de Cúcuta 1821.Tomo II (Bogotá: Fundación Francisco de Paula Santander, 1989), 130.

Actas del Congreso de Cúcuta 1821, 109.

Actas del Congreso de Cúcuta 1821, 124.

Patricia Londoño, "Educación femenina en Colombia, 1780-1880", Boletin Cultural y Bibliográfico Vol: 31, No. 37 (1994), 35. 
$2^{\circ}$. Que sin embargo de las ventajas que ella ofrece no puede plantearse sin crear un fondo para la dotación de la maestra y arrendamiento del local respectivo de las rentas comunales señaladas en el numeral 4 de la ley orgánica de provincias; y que según el tenor de nuestra ley las rentas comerciales deben invertirse con preferencia en el establecimiento de escuelas,

Decreta:

$1^{\circ}$. A la brevedad posible se establecerá en esta ciudad una escuela de niñas cuya maestra será nombrada a juicio de la sociedad de educación, eligiéndose de entre las personas aparentes la de más virtud y respetabilidad.

Son obligaciones de la maestra: enseñar $1^{\circ}$. Las verdades de la Religión comprendidas en la doctrina cristiana: $2^{\circ}$. Las principales máximas de la buena moraly urbanidad: $3^{\circ}$. Leer y escribir: $4^{\circ}$. Coser, bordar y dibujar?.

Hubo especial atención en la selección de la maestra por ser quien iba a garantizar la buena orientación de las niñas. No podía ser cualquier persona, la elegida debía cumplir con algunos requisitos previos satisfactorios con las expectativas normativas de la sociedad, además de "ser la de más virtud y respetabilidad": ser católica, constancia de un comportamiento moral impecable, y además saber leer y escribir. Teniendo en cuenta las características consideradas indispensables para el buen funcionamiento escolar se seleccionó a la señorita Margarita Polo para que asumiera la responsabilidad de ser la primera maestra en la formación de las niñas pastusas.

El nombrar una persona como la señorita Polo con la habilidad de la lectura y de la escritura lleva a suponer que a pesar de no existir una escuela en la ciudad, esto no fue óbice para que algunas jovencitas hubieran logrado esa formación mediante un aprendizaje presumiblemente doméstico, como era costumbre en otras localidades en donde lo realizaban en el sitio considerado el más adecuado para las niñas, como era la casa, generalmente bajo la enseñanza de la madre o de una vecina de la familia ${ }^{10}$.

\footnotetext{
Archivo Histórico Municipal de Pasto (AHMP), Pasto. Correspondencia, Pasto 1835-1837, Caja 14, Folio 55.

10 Para la época fueron comunes en Europa y en algunos países de América las escuelas domésticas de carácter privado conocidas como las Amigas o Migas. Las maestras recibían el mismo nombre del establecimiento, reunían a las niñas en sus propias casas y enseñaban a memorizar el catecismo, coser, tejer, bordar y leer en letra impresa o manuscrita. Pilar Gonzalbo Aizpuru, La educación de la mujer en la Nueva España (México D.F: Secretaría de Educación Pública, Subsecretaría de Cultura, Dirección General de Publicaciones, 1985), 16. Según Pilar Foz y Foz "las Amigas, muy extendidas por la América española, no dejaron huella bajo tal nombre en la Nueva Granada", Pilar Foz y Foz, Mujer y Educación en Colombia. Siglos XVI-XIX (Bogotá: Academia Colombiana de Historia, 1997), 33. Sin embargo, algunas niñas fueron educadas en su casa, como es el caso de las niñas de la élite pastusa.
} 
Fueron cuarenta y cinco años durante los cuales, sin haber recibido preparación especial, un grupo de mujeres trabajaron como maestras de primeras letras de las niñas de la localidad. Su número iba en aumento en la medida en que mejoraba la organización de escuelas para las niñas en toda la región sur del país. Sin embargo, casi al finalizar el siglo XIX algunos personajes de la ciudad, a la vez que reconocían el aporte de las maestras, lamentaban que las pastusas no tuvieran otros campos de acción diferentes al convento y a la escuela, reconociendo que no era porque les faltaran aptitudes, sino porque "la instrucción que les damos es muy reducida, en la extensión de las materias de aprendizaje como en el número de personas favorecidas" ${ }^{11}$ Declaración que admitía la responsabilidad del gobierno, la iglesia católica e intelectuales, con la complicidad de las mismas mujeres, al insistir en mantenerlas, alejadas de las luces, como un personaje "de la galería de fantasmas" según la denominación que le da Belinda Arteaga a la permanente minoría de edad.

\section{Las primeras maestras profesionales}

En América Latina la formación profesional de maestras ocurrió a partir de la segunda mitad del siglo XIX y fue asumida por los gobiernos de cada país, según las circunstancias, dentro del contexto general en el cual las nuevas repúblicas ingresaron a consolidar sus procesos de modernización. Desde esta perspectiva la preparación de maestras en Colombia hizo parte del proyecto educativo del gobierno liderado por los liberales radicales, para quienes el impulso a la instrucción pública era una de las mejores estrategias "para combatir el analfabetismo y hacer de la sociedad colombiana una sociedad ilustrada, ansiosa de progreso" .

En ese contexto, la organización de las Escuelas Normales de Institutoras hizo parte de un proyecto político que pretendió dar respuesta a la necesidad de formar sectores de la población femenina para dar cuenta en la vida cotidiana de una nueva cultura. El gobierno liberal era consciente, del atraso en el que se encontraba la mayoría de la población femenina del país. Un radical como Salvador Camacho Roldán llegó a reconocer "la indolencia que habían tenido los liberales colombianos respecto a la educación femenina" ${ }^{13}$, lo que

11 Alejandro Santander, Biografía de D. Lorenzo de Aldana y Corografía de Pasto (Pasto: Imprenta de Gómez Hermanos, 1896), 98.

12 Myriam Báez Osorio, "Las Escuelas Normales y el cambio educativo en los Estados Unidos de Colombia en el período Radical, 1870-1886" (Tesis Doctorado, Universidad Pedagógica y Tecnológica de Colombia, 2004).

13 Gabriela Hernández Vega, La mujer en la Universidad de Nariño: 1935-1969 (Pasto: Universidad de Nariño, 2004), 103. 
ameritaba el impulso a un proyecto educativo tendiente a crear condiciones para que salieran de esa marginalidad. Según él, entre los liberales la mujer:

Ha estado confinada al hogar doméstico; para ella no han existido universidades ni colegios ni educación avanzada que inicie en su mente las nuevas ideas del siglo. Ella es lo que era antes de la independencia. No hemos tratado de colocarla en la corriente del siglo, y se han quedado atrás de nosotros ${ }^{14}$.

A los liberales les urgía preparar a las mujeres con más ilustración que en épocas anteriores; era necesario actualizar su educación con los nuevos valores que debían desplegar en su rol doméstico como era el de formar al nuevo ciudadano. El proyecto educativo liberal impulsó, aún en contra de quienes se oponían a la idea de proporcionar conocimientos científicos a las jóvenes, la organización de Escuelas Normales para la formación de maestras en todos los Estados de la Unión, lo que representó un avance respecto a la educación femenina ${ }^{15}$ de corte tradicional.

Sin embargo, la modernización de la educación no tuvo ninguna repercusión en la situación política y civil de las mujeres. Para los liberales la formación profesional de maestras, no buscó producir cambios en el pensamiento político o en las definiciones jurídicas respecto a la inequidad femenina. Al contrario, con la constitución política de 1863 y la aprobación del código civil, los liberales institucionalizaron y legalizaron la subordinación de las mujeres dentro del matrimonio a la vez que ratificaron su exclusión de lo público.

En ese ambiente de fraternidad masculina y subordinación femenina apareció la primera Escuela Normal Femenina del país organizada en el Estado Soberano de Cundinamarca, inició labores en la ciudad de Bogotá -capital del país- en 1872. La experiencia de esta institución sirvió para que el gobierno central fomentara a nivel nacional los estudios normalistas para las jóvenes: "al lado de la creación de las Escuelas Normales de varones surgió el interés por la fundación de Escuelas Normales de mujeres, que favoreció la formación de maestras" ${ }^{\prime 16}$.

Con el antecedente de la Normal de Cundinamarca, en 1874 Santiago Pérez, presidente en ese momento de los Estados Unidos de Colombia, expidió el decreto mediante el cual reglamentó lo correspondiente a las

14 Salvador Camacho Roldán, Escritos varios. Tomo II. (Bogotá: Editorial Incunables, 2a . Edición. 1893), 78.

15 Londoño, "Educación femenina en Colombia, 1780-1880", 35.

16 Myriam Báez Osorio, "Las Escuelas Normales y el cambio educativo en los Estados Unidos de Colombia en el período Radical, 1870-1886", 158. 
Escuelas Normales femeninas. Determinó que, como había ocurrido con las Normales de varones, "Se establecerá una Escuela Normal de Institutoras en cada uno de los Estados donde no existe ningún plantel de la misma clase, de conformidad con el presente decreto" ${ }^{17}$; al finalizar la década existían en el país Normales femeninas en Antioquia, Bolívar, Boyacá, Cauca, Cundinamarca, Panamá, Santander, Magdalena y Tolima.

La profesión de maestra pasó a ser reconocida como una actividad propia para el bello sexo, dada la analogía que habían establecido entre "la naturaleza femenina" y la educación formal de las niñas y las jóvenes. Para la sociedad, las maestras lograban conjugar el sentido de ser madre con la capacidad de entrega al otro; existía la creencia de la supuesta capacidad innata de las mujeres para las tareas educativas, para el cuidado moral y material de la niñez. Razón para entender este oficio como una prolongación de la maternidad en lo referente a las "labores domésticas de atención y educación de los niños"18, "al igual que la costura, constituía una prolongación de las actividades que ellas debían realizar en el hogar" 19 . El sentido de la escolaridad para las mujeres se centró en proporcionarles mejores condiciones educativas para "darse a otros, sobre todo a un hombre, nunca para formarse a sí misma, y por tanto no le corresponde el cultivo de la ciencia, ni de la filosofía ni de la poesía"20; se trataba de que la madre de sus hijos no fuera una analfabeta.

Por esas similitudes de la profesión, la dedicación de las mujeres al magisterio fue considerada de orden esencialista; era como si las mujeres hubieran nacido para ser maestras, una interpretación que no permitía evidenciar el origen histórico-social de las vocaciones. Sin embargo, tanto la formación como la dedicación de algunas mujeres al magisterio, fueron de vital importancia para el futuro de la acción en lo público de la población femenina, en cuanto ampliaron el espectro existencial, en este caso, de las colombianas. El magisterio, se convirtió en una actividad a través de la cual algunas jóvenes de Pasto y de la región sur del país lograron dar un paso para superar la marginalidad social en la que permanecían siendo ya las postrimerías del siglo XIX.

17 Decreto Número 356 de 1874 (27 de agosto) Sobre establecimiento de Escuelas normales para mujeres. Artículo $1^{\circ}$.

18 Magdala Velásquez Toro, "Condición jurídica y social de la mujer", en Nueva Historia de Colombia,. Tomo IV (Bogotá: Planeta Colombiana Editorial S. A, 1989), 26.

19 Suzy Bermúdez, El Bello Sexo La mujer y la familia durante el Olimpo Radical. (Santafé de Bogotá D.C: Ediciones Uniandes, Ecoe Ediciones, 1993), 123.

20 Gabriela Castellanos, “¿Existe la mujer? Género, Lenguaje y Cultura”, en Género e Identidad. Ensayos sobre lo masculino y lo femenino, eds. Luz Gabriela Arango et al (Santafé de Bogotá: Tercer Mundo Editores, Ediciones Uniandes, Programa de Estudios de Género, Mujer y Desarrollo, Facultad de Ciencias Humanas, Universidad Nacional de Colombia, 1995), 40. 
Independientemente de las limitaciones y de las dificultades que se presentaron, el desempeño como maestras fue un hecho con beneficios personales y colectivos para las mujeres: "les dio por primera vez la oportunidad de acceder a una profesión respetable, que las colocó en un nivel influyente, otorgándoles simultáneamente una nueva dignidad y la posibilidad de suministrar un negocio rentable"21.

\section{Escuela Normal de Institutoras de Popayán}

La dependencia político-administrativa de Pasto, la provincia de su nombre y demás provincias de la región sur del país con el Estado Soberano del Cauca, fue la razón para que en las últimas décadas del siglo XIX las pastusas, los pastusos o quienes de otras partes de la región aspiraran a realizar estudios normalistas tenían que viajar a Popayán ciudad en la cual, por ser la capital del Estado, se encontraban las Escuelas Normales femenina y masculina.

Para la formación de maestras en el Estado del Cauca, antes de la Normal, existió en Popayán el Colegio de Señoritas organizado por las autoridades locales en respuesta a la preocupación de los padres de familia por tener una institución donde sus hijas pudieran continuar estudios al salir de la escuela primaria. Autorizado por la Ley 39 de 1873, expedida por el gobierno del Estado, funcionó durante cuatro años hasta su transformación en 1877 en Escuela Normal de Institutoras. Fue creado con fondos del Estado destinados exclusivamente para "la educación del bello sexo"; incluyó en su plan de estudios asignaturas correspondientes a la preparación pedagógica por lo que sus estudiantes fueron las primeras maestras especializadas para atender las escuelas de niñas del Estado.

La posibilidad para que algunas jóvenes de Pasto y de la zona sur llegaran a ingresar a la Normal de Popayán fue un avance en la educación femenina del sur del país, pero, a su vez, el número de ingresos adjudicados se convirtió en un obstáculo para formar un grupo suficiente de jovencitas que tuvieran a su cargo las escuelas de la región; a Pasto y demás provincias del sur les correspondió un cupo por promoción lo que significó que cada año solamente una joven de la región podía ingresar, por cuenta del Estado, a la Escuela Normal de Institutoras de Popayán. Situación altamente desfavorable teniendo en cuenta la necesidad de maestras debidamente preparadas que requería la educación de las niñas en cada una de las provincias. A pesar

${ }^{21}$ Aída Martínez Carreño, "Opciones femeninas en la Colombia del siglo XIX”, Gaceta. Edición 6, 56. 
de las dificultades fue posible la conformación de un grupo reducido, pero altamente cualificado, de jóvenes maestras quienes antes de finalizar el siglo XIX impulsaron cambios y lograron imprimir una nueva dinámica a la educación femenina en esta zona del país.

Para la época en la cual organizaron las Escuelas Normales en el país, ya no se discutía la necesidad e importancia respecto a la educación formal de las mujeres, pero no existía consenso respecto a qué enseñarles, hasta ese momento no se había podido conformar una voz unánime acerca del contenido de su educación. La bachillera era incómoda, o como lo expresaba un miembro de la Escuela Literaria de la ciudad de Pasto a propósito del poema escrito por la joven pastusa Dolores Guerrero: "enemigos de la literata, pero amantes y admiradores de la poetisa que distrae sus apacibles ocios" ${ }^{\prime 22}$; educar a las mujeres pero sin salir de los marcos de la puerta de la casa, sin que se refundiera en el mundo de las teorías.

\section{Primeras maestras profesionales}

Por el límite impuesto por el gobierno del Cauca, en las últimas décadas del siglo XIX solamente tres jóvenes provenientes del sur pudieron estudiar en la Escuela Normal de Institutoras de Popayán; al terminar los estudios regresaron a sus respectivos municipios para trabajar en las escuelas oficiales que existían: Amalia Santander, Hortensia Mora y Elena Fernández de Córdoba.

\section{Amalia Santander Villamarín}

Nació en Pasto, en una de las familias de reconocido raigambre liberal; ingresó en 1875 como estudiante en el Colegio de niñas de Popayán donde obtuvo el título correspondiente, convirtiéndose en la primera mujer de la región del sur en realizar estudios normalistas. Parte de los compromisos adquiridos por Amalia como becaria, además de "permanecer en el Colegio de niñas establecido en esta ciudad por el tiempo necesario para hacer los cursos que fija el Reglamento", fue el de "servir por tres años consecutivos en la Escuela para la que fuere nombrada maestra, después de haber concluido el curso en el Colegio"'23.

Una vez graduada, al regresar a la ciudad de Pasto fue nombrada maestra en la escuela de niñas y al año siguiente pasó a ser directora de la institución. Según

22 Dolores Guerrero, "A la señorita Rosario Rodríguez en el día de su natalicio”, El Precursor, Pasto, 15 Octubre, 1887, No 22.

23 Archivo Central del Cauca (ACC), 1875, Paquete 132, leg. 69. 
el Decreto Orgánico de Instrucción Pública -DOIP- las Escuelas Superiores solamente podían estar dirigidas por maestras o maestros normalistas. En consecuencia, los estudios realizados por Amalia abrieron la posibilidad para que las autoridades educativas pudieran tomar la determinación de transformar la Escuela de primeras letras de Niñas de Pasto en una Escuela Superior, lo que, a la vez, dio lugar a un enfoque diferente en el Plan de Estudios para las niñas de la localidad. La Escuela Superior de Niñas funcionó con el Plan previsto en el DOIP, cuya formulación en cuanto a las asignaturas y métodos de enseñanza permitió un avance en la educación femenina en la ciudad.

Doña Amalia se distinguió por la administración que dio a la escuela: bajo su dirección se logró conformar un grupo promedio de 120 niñas que asistían diariamente a clases. Un número bastante alto en comparación con la matrícula y asistencia de las niñas a la escuela en años anteriores, cuando la presencia diaria de las estudiantes llegaba escasamente a veinticinco de las noventa que estaban matriculadas.

El desempeño de Amalia como maestra no tuvo objeciones; sin embargo, por ser la directora de una institución que representaba el pensamiento educativo liberal tuvo que afrontar situaciones bastante conflictivas con el clero y con el gobierno local. En el primer caso se vio en la necesidad de confrontar al Presbítero Joaquín Delgado por su agresión a un grupo de niñas de la Escuela Superior; el jerarca sacó a las niñas de la Iglesia porque asistían a la escuela liberal; el segundo caso fue su enfrentamiento con el Jefe Municipal de Pasto situación que concluyó con su destitución como directora de la Escuela Superior de Niñas.

Durante el desarrollo de la guerra civil de 1885, don José María Navarrete como Jefe Municipal de Pasto solicitó a doña Amalia Santander que presentara su renuncia como directora y maestra de la Escuela Superior de Niñas de esta localidad. El mandatario la acusó de intervenir en política porque con otras señoras habían auxiliado a un grupo de presos políticos entre los cuales se encontraba el liberal Manuel Sarria, antiguo secretario de gobierno del Cauca.

Pasto 2 de mayo de 1885

Jefe municipal don José María Navarrete

Presente

No be impuesto de su atenta esquela, de fecha de ayer, y en contestación digo a usted lo siguiente: 
En circunstancias normales mi deposición necesitaría ser justificada y comprobada la causa según lo establece el artículo 131 del código de I.P.P. para que yo pudiera vindicarme; pero en las anormales que atravieza (sic) el país podrá tal vez. de ponérseme (sic) sin haber otro motivo que la chismografía de algún malqueriente que me haya indispuesto ante usted. Tal es la razón que tengo para no renunciar al destino como usted me lo insinúa porque aquello implicaría la confesión mía de haber cometido alguna falta grave de la cual no me acusa mi conciencia.

Si el haber excitado la caridad de varias señoras de esta ciudad, para servir los alimentos diarios al señor Doctor Manuel Sarria y a otros presos políticos que carecen de los medios de subsistencia según es notorio, ha sido el motivo para que se crea que intervengo en las cosas políticas juzgo muy equivocado que se califique de reprensible mi conducta y enseñarla como la virtud que enaltece á la humanidad.

Por todo manifiesto á usted que si el gobierno del cual es leal y decidido oyente le ordena mi deposición estoy pronta a sufrirla, sin que reconozca la grave causal que se me imputa y hagan aparecer al sexo al que pertenezco fuera del campo de acción que le pertenece en la guerra intestina del país.

Dejo en estos términos contestada su precipitada esquela.

Quedo del señor agente municipal su obsecuente amiga, Amalia Santander ${ }^{24}$.

Para doña Amalia visitar y auxiliar a Sarria en la cárcel era un acto de caridad y de agradecimiento con una persona conocida y quien años atrás le había prestado toda su colaboración y apoyo cuando ella solicitó el ingreso al Colegio de Popayán en 1875. Año en el que el liberal César Conto era Presidente del Estado Soberano del Cauca y "Manuel Sarria desempeñaba el cargo de Secretario de Gobierno del Estado" "25, razón para que tuviera que definir la solicitud de ingreso de Amalia que, en este caso, fue una respuesta favorable. Atender a los presos como muy bien lo expresa ella en la carta, era parte del papel que, desde su punto de vista, debían desempeñar las mujeres en la guerra.

Otra fue la visión e interpretación que dio al hecho el Jefe Municipal de Pasto don José María Navarrete para quien la actuación de la maestra Amalia iba más allá de la caridad y solidaridad humanitarias ya que se trataba de la atención prestada a un enemigo político del gobierno del cual ella era funcionaria. La solicitud de renuncia es un hecho que ocurrió tres meses después de la rendición liberal, pactada entre delegatarios del Jefe Municipal José María Navarrete por parte de los conservadores y la comisión conformada por los representantes del jefe liberal General Rosas, de la cual hacían parte

24 Archivo Histórico Municipal de Pasto (AHMP), Pasto 1884-1885, Caja 10, leg. 17, f. 6.

25 Archivo Central del Cauca (ACC), 1875, leg. 69, f. 132. 
los hermanos Santander. En este contexto, el oficio dirigido a Amalia para que dejara el cargo se entiende como una represalia de los conservadores en cabeza del Jefe Municipal contra una maestra considerada adversaria política.

Vencidos los liberales la respuesta de Amalia no tuvo ninguna audiencia, al contrario, un mes más tarde ya había sido reemplazada por Primitiva Velasco, según lo confirma el oficio que esta maestra envió el 11 de junio del mismo año informando que está al frente de la Escuela oficial de niñas "por entrega de Amalia Santander" ${ }^{26}$. Primitiva no había realizado estudios normalistas pero desde algún tiempo se desempeñaba como maestra en escuelas de la Provincia.

\section{Hortensia Mora}

Considerada una señorita, "de virtudes morales diamantinas y de probadas capacidades intelectuales y técnicas para el cultivo de la mujer"27, no era pastusa de nacimiento ya que su llegada al mundo ocurrió en Túquerres, pero fue la segunda jovencita de la región sur que recibió grado de Maestra de Escuela Superior en la Normal de Institutoras de Popayán en $1881^{28}$.Al igual que lo ocurrido con Amalia en Pasto, el título de Hortensia como normalista superior dio la posibilidad para que en su ciudad natal la educación de las niñas pudiera avanzar mediante la organización de la Escuela Superior de la cual fue directora y que atendió en compañía de su hermana Sofía Mora.

A manera de saludo en la ceremonia de graduación Hortensia dirigió unas breves palabras a los asistentes que, en cierta medida, dejan entrever el valor que ella atribuye a la educación como factor indispensable para la libertad de los pueblos y el avance de la sociedad mediante la instrucción de las mujeres:

\section{Señores:}

Con el objeto de manifestar el elevado sentimiento de gratitud que abriga mi corazón respecto de todos los que han contribuido al coronamiento de mi carrera escolar, tomo la palabra en estos momentos solemnes en que la civilización se presenta en todo su esplendor, regada con el más prestigioso de sus banquetes, á los pueblos y á los gobiernos que han hecho esfuerzos sobrehumanos para conquistarla y disfrutar de sus benéficos resultados. Y es particularmente al del Cauca á quien toca vestirse de gala en este día, porque presencia la apoteosis de su libertad alcanzada, á fuerza

26 Archivo Histórico Municipal de Pasto (AHMP), Pasto 1884-1885, Caja 10, leg. 17 , f. 9.

27 Leónidas Coral, La guerra de los Mil Días en el Sur de Colombia. (Pasto: Editorial Nariño, s.a), 100.

28 El Escolar, Periódico oficial de Instrucción Pública del Estado Soberano del Cauca, Popayán, No 97, 27 Julio, 1882, 1. 
de incesantes luchas en el campo de las letras, de sacrificios inauditos en los campos de batalla y simbolizada en el perfeccionamiento del género humano, mediante la educación e instrucción de la mujer.

Por eso, yo, llena del ardiente entusiasmo que dá é inspira la juventud, y de reconocimiento y de admiración hacia el pueblo de mi patria que tales lauros ha alcanzado en cambio de azarosas faenas y de sangre de sus bijos, reverente me inclino ante él, y le dedico agradecida el diploma de capacidad con que acaba de habilitárseme, aunque sin merecerlo, para dirijir (sic) una escuela. Que acepte mi pequeño óbolo, como lo unico que puedo consagrarle digno de él. Yo por mi parte agotaré mis esfuerzos y mis limitados conocimientos a fin de colocarme á la altura de la misión verdaderamente regeneradora que se me ha confiado. (...)

Permitidme ahora que me dirija en particular a la Directora de este establecimiento y les signifique este testimonio de mi adhesión y de mi sincera gratitud por la parte activa y directa que han tomado en mi educación y por las consideraciones que me han dispensado. Quiero además que sepan que nunca se borrará de mi mente el recuerdo de sus nobles cualidades y que por toda mi vida guiada me sentiré por los sanos consejos y saludables lecciones que me han inculcado.

Por último, vosotras, mis amadas compañeras de estudio, aceptad el sentido adiós que os dirijo y los votos fervientes que al cielo elevo por vuestra felicidad y porque obtengáis al coronar vuestra carrera escolar el éxito lucido que es de esperar de las aptitudes que os acompañan y que acompañan igualmente á nuestras benévolas Directoras. He dicho ${ }^{29}$.

\section{Elena Fernández de Córdoba}

Finalmente una tercera maestra fue Elena Fernández de Córdoba, nació en la ciudad de Ipiales e hizo parte del grupo privilegiado de jovencitas de la región sur que estuvo en condiciones de viajar a Popayán a realizar estudios en la Normal de Institutoras, los que concluyó "titulándose como Maestra Elemental en 1889"30.

\section{Los colegios de las maestras decimonónicas}

Un rasgo distintivo de estas maestras por el que sobresalieron en el siglo XIX, fuera del interés que mostraron por su formación profesional, son todas las acciones que realizaron en favor de la educación de las niñas de la región; esas acciones se convirtieron en testigos directos de su pensamiento. Con

29 Hortensia Mora, "Palabras de despedida en la sesión de distribución de premios de la Escuela Normal de Institutoras", El Escolar, Popayán, 23 agosto, 1882, No. 101, 2.

30 Informe del Ministro de Instrucción Pública al Congreso de 1913. Diplomas de Maestros, expedidos por las Escuelas Normales de la República. (Bogotá: Imprenta Nacional, 1913), 82. 
su pragmatismo demostraron que abogaban por una población femenina ilustrada, no querían mujeres analfabetas. Sabían del valor de la educación para la población femenina y para la sociedad, en particular de sus beneficios para la región, de ahí la constancia al implementar proyectos educativos.

La primera consecuencia de trascendencia social que produjo la formación de las maestras recayó directamente en beneficio de la población femenina infantil. Con estas maestras graduadas se logró ampliar la cobertura de la escolaridad pública así como la cualificación de los procesos pedagógicos mediante la organización en las principales ciudades de la región como Pasto, Ipiales y Túquerres de las escuelas de carácter superior. Las tres poblaciones vivieron la transformación de las escuelas de primeras letras en Escuelas Superiores para las niñas.

Coherente con lo anterior, el segundo impacto tuvo lugar con la organización que hicieron las normalistas de colegios privados para la educación de las adolescentes en Pasto e Ipiales, ésto dio lugar a una verdadera revolución en la cotidianidad de las jovencitas pertenecientes al sector social de buenos recursos económicos. La apertura de los colegios benefició a las jóvenes en dos sentidos: el primero permitió que la enseñanza respondiera a un plan de estudios sistematizado, el segundo fue motivo para que ellas necesariamente tuvieran que salir a diario de su casa para ir al colegio, ésto dio lugar a un cambio en las costumbres de las familias.

En el siglo XIX las jóvenes de Pasto, salvo la asistencia a misa y a oficios religiosos eran escasos los motivos por los que ellas salían a la calle, permanecían en sus casas, hasta tal punto que a finales del siglo los jóvenes pertenecientes a la Escuela Literaria se quejaban respecto a su encierro.

Antes de que existieran los colegios organizados por las normalistas y los de comunidades religiosas las niñas hijas de las familias de mayores recursos económicos realizaban los estudios en sus casas, las clases eran atendidas por profesores privados contratados por los padres; así lo explicaba en su informe el director de Instrucción Pública "la mayor parte de los padres de familia de proporciones tienen maestros rentados, los que van diariamente á las casas á dar lecciones á las niñas" ${ }^{\prime 1}$.

Después de dejar el cargo como directora de la Escuela Superior de Niñas Amalia Santander decidió continuar su ejercicio profesional dedicada

31 Archivo Histórico Municipal de Pasto (AHMP), Correspondencia, Caja 9, leg. 6, f. 9. 
a la educación privada. Varias familias residentes en la ciudad de Ipiales, preocupadas por la educación de sus hijas, decidieron aunar esfuerzos para organizar un colegio femenino que estuviera en condiciones de brindar una buena educación a las jóvenes de la localidad. Esta iniciativa dio lugar a la creación del colegio de carácter privado Nuestra Señora de las Lajas, sin prevenciones de ninguna naturaleza, contrataron a doña Amalia Santander para que dirigiera el nuevo colegio.

No se conoce con exactitud el plan de estudios del colegio de Nuestra de las Lajas, pero sí de la satisfacción y complacencia de los padres de familia por el desempeño pedagógico de la directora. En la sesión solemne de agosto de 1889, que daba por finalizado el año escolar, la Junta Directiva del colegio dio lectura del acta donde los padres dejaban constancia del excelente resultado de los exámenes así como de la "exaltación de las virtudes y talentos de la cultísima hija de Pasto, señorita Santander"32.

Después de permanecer unos pocos años en Ipiales Amalia regresó a Pasto. De nuevo en la ciudad capital con su prima Elena Eloísa Santander decidieron fundar el Colegio de la Concepción para señoritas, el cual inició labores el 1o. de diciembre de 1891 con "Amalia como Directora y Elena

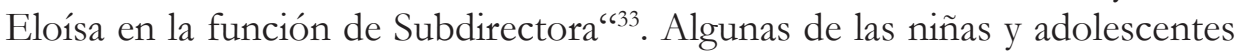
que ingresaron como estudiantes eran de la ciudad y fueron matriculadas en calidad de externas, pero también ingresaron niñas que venían de otras ciudades de la región a quienes aceptaron como estudiantes internas. Por ser una institución de carácter privado los padres de familia debían cancelar una pensión mensual de seis a ocho reales.

La enseñanza estaba bajo la responsabilidad de la Directora y Subdirectora quienes, además de las funciones administrativas, tenían a su cargo diferentes asignaturas correspondientes a los niveles de primaria y secundaria; una excepción era la clase de Dibujo por la que respondía el profesor señor Apolinar Guzmán. Por su calidad académica la prensa local hacía referencia a los "lucidos exámenes presentados por las estudiantes del Colegio de la Inmaculada Concepción, del que son Directoras las señoritas Elena y Amalia Santander" 34 .

El 6 de octubre de 1893 inició labores el Liceo de la Merced dirigido por la señorita Elena Fernández de Córdoba y donde estudiaba, según

\footnotetext{
Leónidas Coral, La guerra de los Mil Días en el Sur de Colombia, 99.

Santander, Biografía de D. Lorenzo de Aldana y Corografía de Pasto, 117.

34 El Bien Público, Pasto, 5 agosto, 1894, Año 1, No. 29, 116.
} 
una nota periodística plena de galanura, "la aristocracia del talento y de la belleza de Pasto"35. La organización del Liceo estuvo "respaldada por los señores Bernardo de la Espriella, Epaminondas Navarrete, comerciantes altamente reconocidos, y por el doctor Manuel María Rodríguez quien fuera director del periódico local El Bien Público"36. Los tres preocupados por la educación de las jóvenes y por el progreso regional eran, a la vez, parte integrante de quienes estaban luchando para que la zona sur del país lograra la independencia del Estado Soberano del Cauca y se pudiera conformar en el décimo departamento.

El plan de estudios del Liceo comprendía básicamente Religión, Lectura, Escritura, Castellano, Aritmética y Geografía, con especial cuidado en el énfasis dedicado a la formación religiosa de las niñas porque, como se ha explicado, para la sociedad pastusa la educación femenina debía tener como fundamento la religión católica: "Una señorita instruida en toda clase de conocimientos de adorno, pero que ha vegetado sin educación sólidamente cristiana, es más perjudicial para con sus padres, esposo é hijos, que de ayuda, consuelo y alegría" 37 .

Sin embargo, en el Liceo dirigido por Elena Fernández de Córdoba, contrario a lo que se podía esperar según los requerimientos de las familias pastusas respecto a la educación de sus hijas, la enseñanza de las asignaturas denominadas propias del sexo no hacían parte del trabajo escolar, un indicio sobre la manera de pensar de la directora de lo que debía ser la formación escolar de las niñas.

A pesar de que uno de los patrocinadores de la institución como era Manuel María Rodríguez, años antes había escrito en El Precursor, periódico de la Escuela Literaria de Pasto, sobre la importancia de la instrucción como "principio de toda regeneración" insistiendo en los aspectos que debía contemplar la educación de la juventud femenina:

atendida la importancia de su ilustración, por la grande influencia que debe ejercer, y la alta misión que está llamada á desempeñar, recibe una esmerada educación moral, ampliamente cientifica, y de exquisito buen tono, por la participación que tienen en ella la literatura, las lenguas, la música, la pintura y las primorosas labores propias del sexo ${ }^{38}$.

\footnotetext{
Erasmo Del Valle, "Liceo de la Merced”, El Bien Público, Pasto, Julio 14, 1894, No 26, 104. Del Valle, "Liceo de la Merced", 23.

Angel De Aviñonet, "La educación de la mujer", El Adalid Católico, Pasto, 2 Junio, 1904, No. 57, 1.

38 Manuel María, Rodríguez, El Precursor, Pasto, 15 mayo 1887, No. 12, 2.
} 
La anotación de Rodríguez hacía eco a la expectativa e interés de los padres de familia, quienes no aceptaban excusa para que en la formación de las niñas no estuvieran presentes las áreas que les iban a permitir lograr una mejor preparación para el desempeño de su función doméstica. Las jóvenes, previo el matrimonio, debían estar en condiciones de dirigir, de administrar correctamente una casa y en especial estar en capacidad de poder hacer un buen manejo del presupuesto familiar. Estos conocimientos y habilidades las mujeres los podían adquirir en sus hogares, pero se esperaba que también hicieran parte de la formación integral que debían impartir los colegios, eran razón de ser del proyecto educativo femenino. En consecuencia, solicitaron a la Directora incluir necesariamente en el plan de estudios la Economía y la Higiene domésticas, que son de incontestable importancia en la educación de la mujer ${ }^{39}$.

El Liceo de La Merced se vio obligado a suspender labores por efectos de la guerra de los Mil Días; doña Elena Fernández de Córdoba y sus hermanas se instalaron en la ciudad de Tulcán ${ }^{40}$.

En la ciudad de Ipiales, después del regreso de Amalia a Pasto, la normalista Hortensia Mora organizara el colegio femenino de carácter privado La Providencia. Doña Hortensia pensó suplir, en parte, la necesidad de preparar maestras para las escuelas de la localidad, por lo que el Plan de Estudios que adoptó era con orientación pedagógica. En este Colegio se graduaron las primeras bachilleres de los Municipios de Obando y Túquerres ${ }^{41}$, quienes llegaron a ser también las primeras pedagogas de escuelas en Ipiales, Túquerres y otros municipios de la Provincia de Obando.

La época en la que funcionó el Colegio La Providencia correspondió al período político de la Regeneración en el cual se cambió el Decreto de Instrucción Pública -DOIP-, que reguló el proyecto educativo de los liberales, por el conjunto de normas que conformaron el proyecto educativo conservador. Sin embargo, para orientar los estudios del colegio doña Hortensia retomó, a pesar de las nuevas directrices del gobierno sobre normales, incrementando un año más en la duración de los estudios, el Plan que ella había seguido durante su formación en la Escuela Normal de Popayán y que correspondía al ideario liberal.

39 Del Valle, "Liceo de la Merced", 104.

40 Es la ciudad ecuatoriana que más directamente limita con Colombia.

${ }^{41}$ Guadalupe Flórez Pérez, Desafío por el futuro. (Ipiales: Ediciones Fundación Antonia Josefina Obando, 2001), 122. 


\section{Colegio de la Providencia}

Plan de Estudios

\begin{tabular}{|ll|}
\hline \multicolumn{1}{|c|}{ Primer Año } & \multicolumn{1}{c|}{ Segundo Año } \\
\hline Religión & Religión \\
Castellano & Física [1er. curso] \\
Aritmética [1er. Curso] & Geometría \\
Geografía general y física & Aritmética [2 ${ }^{\circ}$. curso] \\
Urbanidad & Castellano superior \\
Lectura y escritura & Ortografía \\
Doctrina Cristiana & \\
Tercer Año & Cuarto Año \\
Religión & Historia sagrada \\
Física [2 $2^{\circ}$ curso] & Historia Patria $\left[2^{\circ}\right.$. curso] \\
Cosmografía & Pedagogía [2 $2^{\circ}$ curso] \\
Economía doméstica & Botánica \\
Historia Patria [1er. curso] & Zoología \\
Geografía de Colombia & \\
Pedagogía [1er. curso] & \\
\hline
\end{tabular}

Directora: Hortensia Mora. (ACC, 1875)

\section{CONCLUSIÓN}

Las maestras pastusas decimonónicas fueron expresión de una real solidaridad de género y se distinguieron por su acción a favor de la escolaridad de las jóvenes de la región. En el siglo XX la acción de las maestras pastusas se manifestó de una manera diferente, logrando un mejor posicionamiento en su relación con el conocimiento. Este cambio, no fue pensado ni deseado por las élites políticas, ocurrió porque en el plano de lo social "del entramado de actos de voluntad y de planes resultan procesos que nadie de los implicados había previsto" 42 , como es posible interpretar el avance que propiciaron, durante los años del estudio, las maestras Amalia, Hortensia y Elena en la educación de las niñas de ciudades como Pasto, Ipiales y Túquerres.

La formación profesional de las maestras sirvió para que en el sur las tres jóvenes recibieran una formación científica y pedagógica con la que lograron realizar acciones educativas que trascendieron la formación de las niñas y de las jóvenes de la región, más allá de lo doméstico, pasando a ser "una de las vías para que las mujeres lograran introducir cambios en la relación entre los sexos" $"$.

$\overline{42}$ Norbert Elias, El proceso de la civilización. Investigaciones sociogenéticas y psicogenéticas (México: Fondo de Cultura Económica, Reimpresión Colombiana, 1997), 151.

43 Pierre Bourdieu, La dominación masculina (Barcelona: Editorial Anagrama S.A., 2000), 109. 
Los colegios que organizaron Amalia y Hortensia, además de atender la propuesta de plan de estudios dada por el proyecto educativo, estas maestras buscaron preparar a las jóvenes en el campo pedagógico, de esta forma incrementaron el número de maestras profesionales que sirvieron en las escuelas de la región, de tal manera que lograron superar el vacío, en cuanto a maestras profesionales, que produjo la política de distribución de cupos dada por el gobierno del estado Soberano del Cauca.

En conjunto, los colegios que organizaron estas maestras, así como el avance en la educación básica de las niñas, logró un cambio cultural frente a la educación de las niñas y de las jóvenes en cuanto al sentido de su ser social, al procurar que un buen número de jóvenes de la región lograr una vinculación laboral como maestras profesionales, además, la preparación de la población femenina consideró, sin dejar la formación en religión-moral y urbanidad, un espectro más amplio al incluir la preparación pedagógica, e introducir a las jóvenes en el estudio de las ciencias naturales y de las ciencias sociales, desde esta perspectiva las maestras buscaban formación de niñas y jovencitas ilustradas.

\section{FUENTES}

\section{Archivos}

Archivo Central del Cauca

Archivo Histórico Municipal de Pasto

\section{Informes}

Informe del Ministro de Instrucción Pública al Congreso de 1913. Diplomas de Maestros, expedidos por las Escuelas Normales de la República. Bogotá, Imprenta Nacional.

\section{Documentos Nacionales}

Decreto Número 356 de 1874 (27 de agosto) sobre establecimiento de Escuelas normales para mujeres.

Ley 39 de 1873 ( $1^{\circ}$. de octubre) sobre creación de un colegio de niñas en Popayán.

\section{Prensa}

A... El Precursor. Pasto, 15 mayo, 1887, No 12.

De Aviñonet, Angel. "La educación de la mujer", El Adalid Católico. Pasto, Junio 2, 1904, No. 57.

Del Valle, Erasmo. “Liceo de la Merced”, El Bien Público. Pasto, Julio 14, 1894, No 26.

"El talento de la mujer es apropiado para las ciencias exactas", El Bien Público, Pasto, 10 marzo, 1894, Número 11.

El Bien Público. Pasto, 5 agosto, 1894, Año 1, No 29. 
El Escolar. Periódico oficial de Instrucción Pública del Estado Soberano del Cauca. Popayán, No 97, 27 Julio, 1882.

Guerrero, Dolores. "A la señorita Rosario Rodríguez en el día de su natalicio", El Precursor. Pasto, 15 Octubre, No 22, (1887).

M.A.O. "Exámenes en el Colegio de las R: R: M: M: Bethlemitas", El Bien Público. Pasto, 5 agosto, No 29. (1894).

Rodríguez, Manuel María. (a) "Pro-Patria”, El Precursor, Pasto, 15 enero, 1887, No 4o.

\section{REFERENCIAS}

Báez Osorio, Myriam. "Las Escuelas Normales y el cambio educativo en los Estados Unidos de Colombia en el período Radical, 1870-1886". Tesis Doctorado en, Universidad Pedagógica y Tecnológica de Colombia, 2004.

Bermúdez, Susy. "Familias y hogares en Colombia durante el siglo XIX y comienzos del XX", en Las Mujeres en la Historia de Colombia. Tomo II. Mujeres y sociedad, Consejería presidencial para la política social, Presidencia de la República, Santafé de Bogotá: Grupo Editorial Norma, 1995.

Bourdieu, Pierre. La dominación masculina. Barcelona: Editorial Anagrama S.A. 2000.

Camacho Roldán, Salvador. Escritos varios. Tomo II. Bogotá, Editorial Incunables, 2ª Edición, 1893.

Castellanos, Gabriela. “¿Existe la mujer? Género, Lenguaje y Cultura”, en Género e Identidad. Ensayos sobre lo masculino y lo femenino, eds. Luz Gabriela Arango et al, Santafé de Bogotá: Tercer Mundo Editores, Ediciones Uniandes, Programa de Estudios de Género, Mujer y Desarrollo, Facultad de Ciencias Humanas, Universidad Nacional de Colombia, 1995.

Coral, Leonidas. La guerra de los Mil Días en el Sur de Colombia. Pasto: Editorial Nariño, s.a.

Elias, Norbert. El proceso de la civilización. Investigaciones sociogenéticas y psicogenéticas. México: Fondo de Cultura Económica, Reimpresión Colombiana, 1997.

Flórez Pérez, Guadalupe. Desafio por el futuro. Ipiales: Ediciones Fundación Antonia Josefina Obando, 2001.

Foz y Foz, Pilar. Mujery Educación en Colombia. Siglos XVI-XIX. Bogotá: Academia Colombiana de Historia, 1997.

Gonzalbo Aizpuru, Pilar. La educación de la mujer en la Nueva España. México D.F., Secretaría de Educación Pública, Subsecretaría de Cultura, Dirección General de Publicaciones, 1985.

Hernández Vega, Gabriela. La mujer en la Universidad de Nariño: 1935-1969. Pasto: Universidad de Nariño, Sistema de Investigaciones, 2004.

Londoño, Patricia. "Educación femenina en Colombia, 1780-1880". Boletín Cultural y Bibliográfico, Vol 31 No. 37, (1994): 35.

Martínez Carreño, Aída. Mujeres y familia en el siglo XIX 1819-1899, en: Las Mujeres en la Historia de Colombia. Tomo II. Mujeres y sociedad. Santafé de Bogotá, Consejería presidencial para la política social, Presidencia de la República de Colombia, Grupo Editorial Norma, (1995): 
Martínez Carreño, Aída. “Opciones femeninas en la Colombia del siglo XIX”, Gaceta. Edición 6, 56.

Molina Petit, Cristina. Dialéctica feminista de la Ilustración. Barcelona: Anthropos, Madrid, Comunidad de Madrid. Consejería de Educación. Dirección General de la Mujer. 1994.

Santander, Alejandro. Biografía de D. Lorenzo de Aldana y Corografía de Pasto. Pasto: Imprenta de Gómez Hermanos, 1896.

Velásquez Toro, Magdala. Condición jurídica y social de la mujer, en: Nueva Historia de Colombia. Tomo IV. Bogotá D.E: Planeta Colombiana Editorial S.A, 1989.

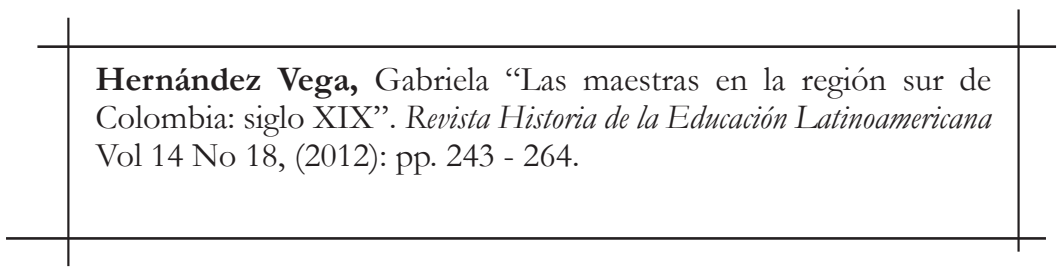

